\title{
Violência obstétrica na assistência ao parto vaginal: uma revisão integrativa entre os anos de 2010 e 2020
}

Obstetric violence in assistance in vaginal delivery: an integrative review between 2010 and 2020

Violencia obstétrica en la asistencia en el parto vaginal: una revisión integrativa entre 2010 y 2020

Aline Gonçalves Gomes ${ }^{1}$, Ângela Mille Ribeiro de Araújo ${ }^{1 *}$, Jeniffer Santos da Silva ${ }^{1}$, Camila Monique Souza de Oliveira Aramaio'.

\section{RESUMO}

Objetivo: Analisar através de artigos científicos, intercorrências de violência obstétrica no parto vaginal no período de 2010 a 2020. Métodos: Utilizou-se como caminho metodológico, uma revisão integrativa da literatura, exploratória de abordagem qualitativa, a partir das publicações nos periódicos da Biblioteca Eletrônica Científica Online (Scielo) no período de 2010 a 2020. Foram encontradas 88 publicações sobre a temática, utilizando os descritores "violência obstétrica" e "violência no parto" sendo 39 (violência obstétrica) e 49 (violência no parto), após a utilização dos fatores de exclusão foram inclusos 15 artigos para serem analisados. Resultados: Ao analisar os artigos selecionados, constatou-se a ocorrência da realização de procedimentos desnecessários, bem como sem autorização, negligência, abuso físico e emocional durante o parto, entre outros. Considerações finais: Entende-se, que todo procedimento realizado sem a autorização da mulher ou sem previsão legal, bem como desrespeito aos direitos e garantias, caracteriza prática de violência obstétrica. Sendo imprescindível, a aplicação de politicas públicas que visem a extinção da violência obstétrica, posto os traumas e consequências que a mesma acarreta.

Palavras-chave: Assistência, Violência, Parto, Obstetrícia.

\begin{abstract}
Objective: To analyze, through scientific articles, complications of obstetric violence in vaginal birth from 2010 to 2020. Methods: An integrative literature review, qualitative and exploratory research, based on publications in the journals of the Online Scientific Electronic Library (Scielo), in the period from 2010 to 2020, was used. Using the descriptors "obstetric violence" and "violence in childbirth" being 39 (obstetric violence) and 49 (violence in childbirth), after using the exclusion factors, 15 articles were included to be analyzed. Results: When analyzing the selected articles, it was found -if the occurrence of unnecessary procedures, as well as without authorization, negligence, physical and emotional abuse during the party, among others. Final considerations: It is understood that any procedure performed without the woman's authorization or without legal provision, as well as disrespect for rights and guarantees, characterizes the practice of obstetric violence. It is essential to apply public policies aimed at the extinction of obstetric violence, given the traumas and consequences that it entails.
\end{abstract}

Key words: Assistance, Violence, Childbirth, Obstetrics.

\footnotetext{
${ }^{1}$ Centro Universitário Aparício Carvalho (FIMCA), Porto Velho - RO.

*E-mail: Angelamille79@gmail.com
}

SUBMETIDO EM: 11/2021 


\section{RESUMEN}

Objetivo: Analizar, a través de artículos científicos, las complicaciones de la violencia obstétrica en el parto vaginal en el período de 2010 a 2020. Métodos: Se utilizó como enfoque metodológico una revisión integradora de la literatura, exploratoria con enfoque cualitativo, a partir de publicaciones en las revistas de la Scientific Electronic Library Online (Scielo) de 2010 a 2020. Se encontraron 88 publicaciones sobre el tema, utilizando los descriptores "violencia obstétrica" y "violencia en el parto", siendo 39 (violencia obstétrica) y 49 (violencia en el parto), luego de utilizar los factores de exclusión, se incluyeron 15 artículos para ser analizados. Resultados: Al analizar los artículos seleccionados, se encontró la ocurrencia de procedimientos innecesarios, así como procedimientos no autorizados, negligencia, maltrato físico y emocional durante el parto, entre otros. Consideraciones finales: Se entiende que cualquier procedimiento realizado sin la autorización de la mujer o sin disposición legal, así como la falta de respeto a los derechos y garantías, caracteriza la práctica de la violencia obstétrica. Es fundamental aplicar políticas públicas orientadas a la extinción de la violencia obstétrica, dados los traumas y consecuencias que conlleva.

Palabras clave: Asistencia, Violencia, Parto, Obstetricia.

\section{INTRODUÇÃO}

Resumidamente, pode-se dizer que a violência obstétrica corresponde aos atos ou condutas baseadas no gênero, resultando morte, prejuízo ou sofrimento de ordem física, sexual ou psicológica. É uma terminologia utilizada para designar ou descrever e se referir a todas as formas de violência que tem origem na assistência ao ciclo gravídico puerperal (TESSER CD, et al., 2015).

O parto vaginal é tido como um momento único e importante, e as parturientes pretendem vivenciar momentos naturais e saudáveis para elas e seus filhos. No entanto, esse parto é considerado um procedimento doloroso, ainda que em um curto tempo, o nível da dor é maior que o aguardado, esse procedimento doloroso e impremeditado tem o aumento da dor com a administração da ocitocina para aceleração do parto (CAVALHEIRO EAM, et al., 2021).

No Brasil, de acordo com dados do Departamento de Informática do Sistema Único de Saúde (DATASUS), o número de operações cesarianas é considerado alarmante levando em consideração as recomendações da Organização Mundial da Saúde (ZANARDO GLP, et al., 2017). Segundo Leal MC, et al. (2014), a maioria das intervenções são consideradas excessivas e sem respaldo científico, associadas aos riscos e complicações sendo consideradas como uma das formas de violência no parto.

Conforme Tesser CD, et al. (2015), a expressão "Violência Obstétrica" (VO) é utilizada para descrever e agrupar diferentes formas de violência (e danos) durante a assistência obstétrica profissional. Incluindo entre eles: agressões físicas, psicológicas e verbais, bem como procedimentos desnecessários e lesivos como: episiotomias, restrição ao leito no período pré-parto, enema, tricotomia e (quase) ocitocina de rotina, ausência de acompanhante, entre os quais se destacam o excesso de cesáreas, crescendo no Brasil há décadas, apesar de haver iniciativas governamentais nesse sentido.

A Organização Mundial da Saúde (OMS) publicou em 1985 recomendações para assistência ao parto e a partir de 1996 defendem a legitimação da participação das mulheres nas decisões acerca do parto (SENS MM e STAMM AMNF, 2019).

Para os autores Andrade BP e Aggio CM (2014), é indiscutível interromper esse processo natural do parto de uma mulher, pois inegavelmente o parto é um momento único e inesquecível na vida da mulher. Diferentemente de outros eventos que requerem atendimento hospitalar, o processo de parto é fisiológico e normal, na maioria das vezes requer apenas suporte, cuidado, atenção e, o mais importante, humanização.

A violência obstétrica afeta diversas mulheres no momento do parto devido a discriminações de gênero, raça ou etnia, socioeconômica, geração ou orientação sexual. Pesquisa realizada pela Fundação Perseu 
Abramo, no Brasil, em 2010, constatou que $25 \%$ das mulheres se declararam vítimas de maus-tratos, prática que costuma ser naturalizada por profissionais e instituições de enfermagem (MENEZES FR, et al., 2019).

Segundo Andrade PON, et al. (2016), O Brasil avançou muito no comprimento de boas práticas, mas o atual modelo de atenção ao parto é marcado pela medicalização da assistência, que se transformaram em intervenções obstétricas rotineiras e desnecessárias, na qual deveriam ser realizadas somente conforme 0 andamento do parto, ações evidenciadas cientificamente.

O presente artigo teve como objetivo realizar uma revisão integrativa dos estudos sobre a situação de violência obstétrica no parto vaginal, assim reconhecendo a condição vivenciada por parturientes que sofreram alguma intervenção desnecessária, ofensas ditas pelos profissionais, ou até mesmo situações que causaram desconforto durante seu processo de trabalho de parto.

\section{MÉTODOS}

A metodologia corresponde a um estudo bibliográfico, do tipo revisão integrativa esta é a abordagem metodológica mais ampla envolvendo revisão, que pode ser incorporada em pesquisas experimentais e não experimentais para a compreensão completa do fenômeno em análise. Inclui também dados da literatura teórica e empírica, além de uma ampla gama de usos: definições conceituais, revisões teóricas e de evidências e análise de problemas metodológicos de assuntos específicos (SOUZA MT, et al., 2010).

Na medida em que a quantidade e a complexidade da informação na área da saúde não param de crescer, torna-se imprescindível o desenvolvimento de competências no contexto da pesquisa científica, para poder definir etapas metodológicas mais concisas e proporcionar aos profissionais um melhor aproveitamento do que foi esclarecido em numerosos estudos e evidências. Nesse caso, a revisão abrangente surge como um método que fornece a síntese do conhecimento e a aplicabilidade de resultados de pesquisas importantes na prática (SOUZA MT, et al., 2010).

Deste modo, tem-se como pergunta norteadora: "Quais os resultados científicos disponíveis na literatura brasileira sobre violência obstétrica no parto vaginal?".

O levantamento bibliográfico foi efetuado nas bases de dados da Biblioteca Eletrônica Científica Online (SCIELO), o que permitiu a busca por estudos publicados no cenário nacional. Sendo que para a seleção dos estudos, optou-se pela inclusão de artigos científicos disponíveis na íntegra, em livre acesso, publicados no período de 2010 a 2020, no idioma português, bem como, aqueles que após leitura do título e resumo, abrangessem aspectos relacionados à temática do estudo.

Os estudos elegíveis foram lidos integralmente, a fim de incluir ou não, aqueles que conseguissem responder à questão norteadora da referida pesquisa. Segue na Figura 1, o fluxograma dos artigos pesquisados. 
Figura 1 - Fluxograma das etapas para a seleção dos artigos desta revisão integrativa.

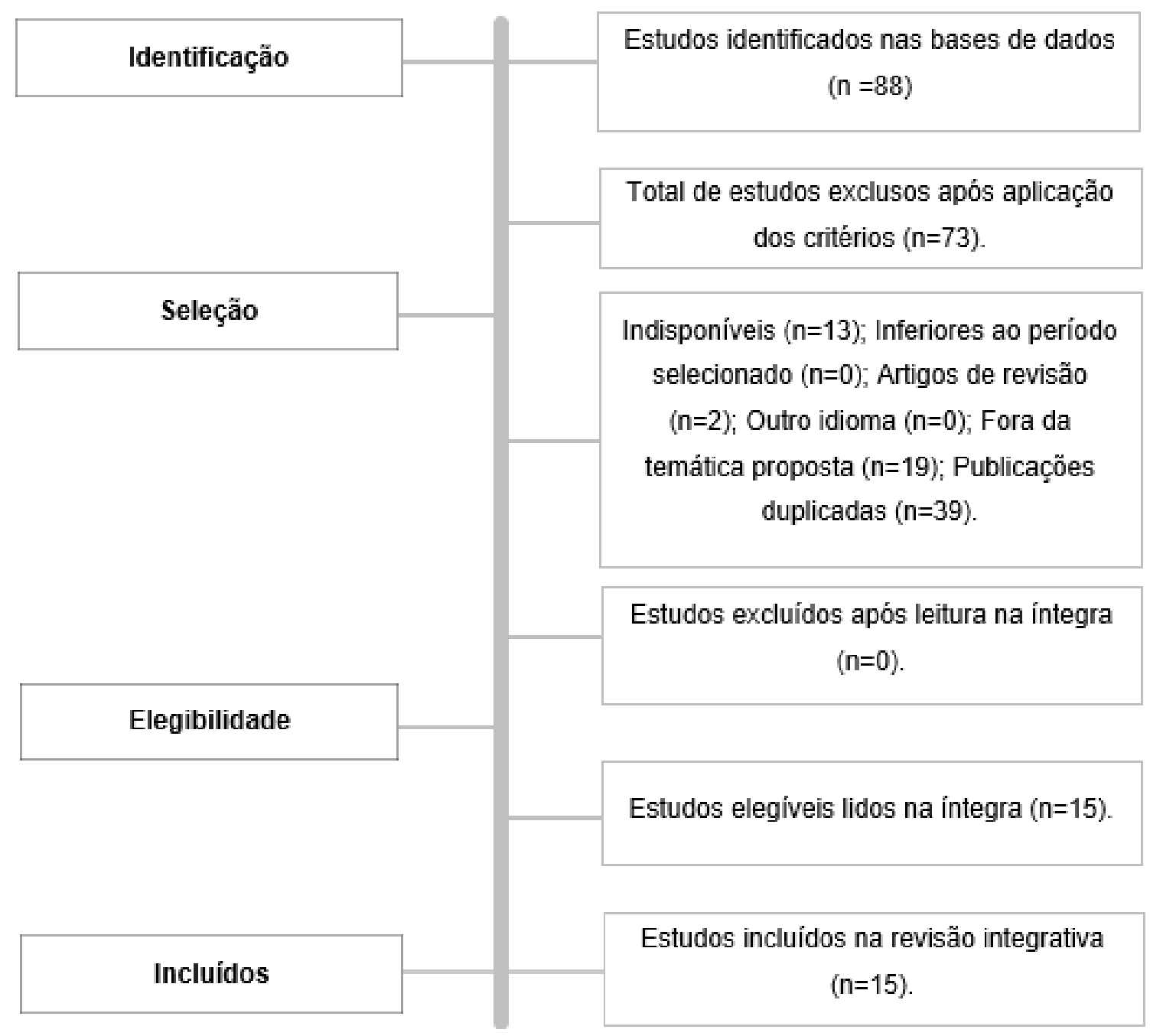

Fonte: Gomes AG, et al., 2021.

Foram encontrados 88 (oitenta e oito) artigos nas bases de dados, dos quais em um primeiro momento, foram exclusos 13 (treze) estudos por estarem indisponíveis, 2 (dois) por serem artigos de revisão, 39 (trinta e nove) por estarem duplicados e 19 (dezenove) fora da temática proposta. Após a leitura do título e resumo, 15 (quinze) artigos foram selecionados para leitura na íntegra, o que possibilitou ao final, a inclusão de 15 (quinze) artigos para serem analisados a fim de subsidiar a revisão de literatura.

\section{RESULTADOS E DISCUSSÃO}

Na realização de buscas nas bases de dados pesquisando a descrição "violência obstétrica" e "violência no parto" na SCIELO, localizou-se 88 (oitenta e oito) artigos elegíveis, no entanto, realizou-se a exclusão de 73 (setenta e três) artigos, por não atenderem os critérios de inclusão ou por não estarem relacionados à temática da presente pesquisa. Dessa forma, o número final de artigos elegíveis foram 15 (quinze). A interpretação e síntese dos resultados encontrados estão demonstrados no Quadro 1 contendo: título, autor, ano, metodologia, bem como as bases de dados de publicação, sobre a violência obstétrica no parto vaginal. 
Quadro 1 - Características dos artigos inclusos neste estudo de acordo com título, autores, ano, metodologia e bases de dados dos artigos analisados.

\begin{tabular}{|c|c|c|}
\hline Autor/Ano & Título & Metodologia \\
\hline $\begin{array}{l}\text { ANDRADE PON, et al. } \\
\text { (2016). }\end{array}$ & $\begin{array}{l}\text { Fatores associados à violência obstétrica na } \\
\text { assistência ao parto vaginal em uma } \\
\text { maternidade de alta complexidade em } \\
\text { Recife, Pernambuco. }\end{array}$ & $\begin{array}{l}\text { Estudo transversal } \\
\text { prospectiva. }\end{array}$ \\
\hline MARTINS AC, et al. (2016). & $\begin{array}{l}\text { Parirás na dor? Revisão integrativa da } \\
\text { violência obstétrica em unidades públicas } \\
\text { brasileiras. }\end{array}$ & Revisão integrativa. \\
\hline SENA LM, et al. (2017) & $\begin{array}{l}\text { Violência obstétrica no Brasil e o } \\
\text { ciberativismo de mulheres mães: relato de } \\
\text { duas experiências. }\end{array}$ & Relato de caso. \\
\hline PALHARIM LA (2017). & $\begin{array}{l}\text { Autonomia para quem? O discurso médico } \\
\text { hegemônico sobre a violência obstétrica no } \\
\text { Brasil. }\end{array}$ & Revisão Integrativa. \\
\hline $\begin{array}{l}\text { ZANARDO GLT, et al. } \\
(2017)\end{array}$ & $\begin{array}{l}\text { Violência obstétrica no Brasil: uma revisão } \\
\text { narrativa. }\end{array}$ & Estudo bibliográfico. \\
\hline $\begin{array}{l}\text { OLIVEIRA VJ e PENNA } \\
\text { CMM (2017). }\end{array}$ & $\begin{array}{l}\text { O discurso da violência obstétrica na voz das } \\
\text { mulheres e dos profissionais de saúde. }\end{array}$ & $\begin{array}{l}\text { Estudo interpretativo com } \\
\text { abordagem qualitativa. }\end{array}$ \\
\hline LANSKY S, et al. (2017). & $\begin{array}{l}\text { Violência Obstétrica: influência da Exposição } \\
\text { Sentidos do Nascer na vivência das } \\
\text { gestantes. }\end{array}$ & $\begin{array}{l}\text { Estudo transversal } \\
\text { multicêntrico e } \\
\text { multimétodos. }\end{array}$ \\
\hline $\begin{array}{l}\text { GUIMARÃES LBE, et al. } \\
\text { (2018). }\end{array}$ & $\begin{array}{l}\text { Violência Obstétrica em maternidades } \\
\text { públicas do estado do Tocantins. }\end{array}$ & Relato de caso. \\
\hline $\begin{array}{l}\text { JARDIM DMB e MODENA } \\
\text { CM (2018). }\end{array}$ & $\begin{array}{l}\text { A violência obstétrica no cotidiano } \\
\text { assistencial e suas características. }\end{array}$ & $\begin{array}{l}\text { Estudo bibliográfico de } \\
\text { natureza quantitativa e } \\
\text { qualitativa. }\end{array}$ \\
\hline ASSIS JF (2018). & $\begin{array}{l}\text { Interseccionalidade, racismo institucional e } \\
\text { direitos humanos: compreensões à violência } \\
\text { obstétrica. }\end{array}$ & $\begin{array}{l}\text { Estudo bibliográfico de } \\
\text { natureza qualitativa. }\end{array}$ \\
\hline SAMPAIO J, et al. (2019). & $\begin{array}{l}\text { Um corte na alma: como parturientes e } \\
\text { doulas significam a violência obstétrica que } \\
\text { experienciam. }\end{array}$ & $\begin{array}{l}\text { Estudo narrativo, com } \\
\text { natureza qualitativa, } \\
\text { interpretativa. }\end{array}$ \\
\hline $\begin{array}{c}\text { MARQUES GM e } \\
\text { NASCIMENTO DZ (2019). }\end{array}$ & $\begin{array}{l}\text { Alternativas que contribuem para a redução } \\
\text { da violência obstétrica. }\end{array}$ & Estudo narrativo. \\
\hline RUSSO JA, et al. (2020). & $\begin{array}{l}\text { Parindo no paraíso: parto humanizado, } \\
\text { ocitocina e a produção corporal de uma nova } \\
\text { maternidade. }\end{array}$ & $\begin{array}{l}\text { Estudo bibliográfico de } \\
\text { natureza qualitativa. }\end{array}$ \\
\hline RIBEIRO DO, et al. (2020). & $\begin{array}{l}\text { A violência obstétrica na percepção das } \\
\text { multíparas. }\end{array}$ & $\begin{array}{l}\text { Estudo qualitativo, de } \\
\text { caráter descritivo, } \\
\text { exploratório. }\end{array}$ \\
\hline PAULA E, et al. (2020). & $\begin{array}{l}\text { Violência obstétrica e } 0 \text { atual modelo } \\
\text { obstétrico, na percepção dos gestores em } \\
\text { saúde. }\end{array}$ & $\begin{array}{l}\text { Pesquisa descritiva, } \\
\text { exploratória, com } \\
\text { abordagem qualitativa. }\end{array}$ \\
\hline
\end{tabular}

Fonte: Gomes AG, et al., 2021.

Posteriormente a leitura e estudo dos artigos selecionados, pode-se observar as seguintes classes de resposta que nortearam o estudo dos dados encontrados: métodos desnecessários e sem autorização, intervencionismo e negligência durante o parto, abuso físico e emocional.

Atualmente, o parto tem sido visto como um método de caráter patológico, que utiliza da tecnologia do parto dirigido, onde a parturiente está, normalmente, semi-imobilizada, com os membros inferiores abertos para cima, sem a realização de consumo de líquidos e alimentos, sujeita a utilização de medicamentos para técnica de indução do parto e utilização constante de episotomia e eventual do fórceps. Sendo está a forma de assistência parto vaginal mais frequente no Brasil, sendo executada normalmente por um profissional 
médico, em um serviço hospitalar, motivo pelo qual também é chamado de parto normal hospitalar (MEDEIROS NCM, et al., 2016).

Até esse momento, tanto a mídia tradicional como a própria sociedade, principalmente, as utilizadoras dos serviços de saúde, indagavam ativistas, profissionais da saúde e investigadoras quanto ao que realmente compreendia a expressão violência obstétrica. Estimuladas por esses questionamentos e com a finalidade de incentivar parturientes a analisar a qualidade da assistência ao parto oferecido, ativista pela humanização do parto pelo Brasil, projetaram e proporcionaram, em 2012, uma pesquisa informal para o qual denominaram de "Teste da Violência Obstétrica" (SENA LM e TESSER CD, 2017).

Esse teste foi disponibilizado online em 8 de março de 2012, ao mesmo tempo em mais de 70 blogs, compreendido como uma avaliação, das mulheres, dos atendimentos oferecidos no decorrer da internação para o parto e nascimento nas maternidades. Em três dias o teste obteve mais de mil respostas, permanecendo aberto por 38 (trinta e oito) dias e neste curto período, avaliou-se um total de 1966 (mil novecentos e sessenta e seis) nascimentos. Apesar de se tratar de uma forma informal, o teste foi usado para incentivar a discussão sobre a violência obstétrica, em conjunto com as mulheres e distintos setores da promoção e atenção à saúde da mulher (PALHARINI LA, 2017).

O profissional absorvido pela realização pode cooperar nos atos e executar os procedimentos sem a autorização da parturiente e não notar que corresponde a atos de violência. Não somente os profissionais podem não ser capazes de identificar os atos de violência, como as mulheres também, visto que não possuem conhecimento fundamental para distinguir os fatos (LEAL MC, et al., 2018).

As participantes apontaram que a falta de atendimento na maternidade é um desrespeito à mãe, o que é considerado uma forma de violência obstétrica. Essa percepção dos gestores é um agravante no campo do parto, pois o acolhimento é uma das diretrizes das políticas públicas para proporcionar às mulheres uma garantia de atenção integral (PAULA E, et al., 2020).

No Brasil, a instituição do Programa de Humanização do Pré-Natal e Nascimento (PHPN), em 2000, objetivava solucionar os pedidos sociais pela melhoria da qualidade de atendimento ao parto e nascimento, tema que tem sido foco de atenção em diversas localidades do país. A finalidade era permitir uma forte discussão quanto ao assunto (RIBEIRO KG, et al., 2021).

Segundo Ribeiro KG, et al. (2021), nessa discussão, o mencionado método do programa é resultado de uma ação de cunho internacional, que iniciou acerca de 30 (trinta) anos, contrários a utilização de formas invasivas, consideradas irracionais e maléficas, no decorrer do parto, buscando favorecer a qualidade da relação mulher/usuária dos serviços de saúde e o profissional da saúde.

O PHPN foi estabelecido pelo Ministério da Saúde, por meio da Portaria/GM n. 569, de 01 de junho de 2000 (MINISTÉRIO DA SAÚDE, 2000). Segundo Ribeiro DO, et al. (2020), em publicação oficial de 2017, foi enfatizado que a mulher pronta para conceber precisa ser respeitada, precisa obter informações baseadas em evidências e participar da tomada de decisões. Portanto, os profissionais que os atendem precisam estabelecer com eles uma relação de confiança e questionar sobre seus interesses e opiniões. Envolver as mães nas decisões sobre seus corpos significa participar ativamente e esclarecer-Ihes como podem ajudar no processo de promoção do parto, em vez de amedrontá-las.

Martins AC e Barros GM (2016), afirmam que no Sistema Único de Saúde (SUS), a violência obstétrica começa em nível institucional. A violência institucional é caracterizada pela carência de acesso das mulheres pobres a serviços essenciais e a vagueação das parturientes em distintas maternidades para serem atendidas.

A perspectiva das mulheres quanto à violência institucional no parto está ligada à falta de qualidade na assistência, evidenciando-se pela falta de vínculo e comunicação entre os profissionais e pacientes, seus familiares e outros profissionais, problemas no diagnóstico médico, falta de acolhimento e resolutividade (GUIMARÃES LBE, et al., 2018). 
Quando o atendimento na unidade de saúde é garantido, as mulheres podem enfrentar a falta de estrutura adequada, força física e recursos humanos instáveis e serem vítimas do processo institucional, que muitas vezes não pode garantir um anestesista de plantão. Aplica analgesia ao parto, sendo vedado 0 acompanhamento durante o parto, embora seja um direito garantido pela Lei n. 11.108 / 2005, ou seja, proibindo o homem de entrar na sala de pré-natal, alegando a falta de espaço físico para garantir a privacidade de outras pacientes, ou a falta de condições particulares de atendimento (BRASIL, 2005; MARTINS AC e BARROS GM, 2016).

A não identificação dos profissionais, a ausência de informação ou informação negada, fragmentada ou confusa, ausência de vínculos entre os serviços que realizam o pré-natal e a maternidade, o descumprimento da lei do acompanhante, a restrição de acompanhante de escolha, a necessidade de intervenção do acompanhante durante o processo de parto, a percepção de que a violência institucional ocorre ainda mais em serviços públicos, a inadequação do ambiente e a precariedade das maternidades, são pontos negativos e constantes nos relatos de violência obstétrica (GUIMARÃES LBE, et al., 2018).

Pelo exposto, observa-se que são necessárias mudanças no cenário brasileiro quanto a temática da violência obstétrica, visto o despreparo, desleixo e imperícia na prática de profissionais da obstetrícia, sejam eles obstetras, técnicos de enfermagem, enfermeiros, entre outros. Sendo que a violência não está relacionada somente ao ato do profissional, mas inicia com o desrespeito do ambiente, a falta de acolhimento ou da assistência sem profissionais qualificados. O investimento constante na formação dos profissionais é fundamental para uma assistência obstétrica de qualidade (ZANARDO GLP, et al., 2017).

Insta desta maneira, que a atenção esteja centrada na mulher e que o serviço reorganize sua conduta acrescentando mecanismos de conscientização de profissionais e ainda, conduza o atendimento da saúde no nível interpessoal a modo que nota a parturiente de maneira holística (HAMMED W e AVAN BI, 2018).

Segundo a OMS (2014), o parto é um procedimento natural que não necessita de controle, mas de cuidados assistenciais, fundamentando-se neste entendimento, a mesma recomenda um envolvimento mais acentuado do enfermeiro obstetra na assistência ao processo de parto e parto, baseado na concepção de que a formação deste profissional é voltada para o cuidado e não para a intervenção.

A diminuição da violência obstétrica e a humanização da assistência hospitalar o parto no Brasil é um problema antigo. O "Projeto Parto Adequado" e o "Projeto ApiceOn" são umas das formas instituídas no Brasil que fundamentam a humanização do parto, além de capacitar os serviços e os profissionais que trabalham no cuidado da gestação, parto e puerpério. No fim do piloto do Projeto Parto Adequado em 2016, o número de partos vaginais em 26 (vinte e seis) hospitais que participaram, teve um crescimento médio e $76 \%$ (setenta e seis por cento), considerando que compreendia a 21\% (vinte e um por cento) em 2014 (MARQUES GM e NASCIMENTO DZ, 2019).

Para que seja possível essa percepção por parte dos profissionais, o uso de instrumentos que avaliem as perspectivas, o entendimento e, por fim, o contentamento com o parto é outra forma que complementa a mulher como um todo. Entender as perspectivas das parturientes é uma forma singular, visto que cada mulher é ímpar, logo, tem sentimentos e incertezas diferentes. A diminuição da violência obstétrica é um desafio na América Latina, entretanto, com o movimento da humanização na atenção à saúde materno-infantil há expectativas quanto ao surgimento de um novo cenário (MARQUES GM e NASCIMENTO DZ, 2019).

Segundo Jardim DMB e Modena CM (2018) acredita-se que a implantação de ações de prevenção e enfrentamento da violência obstétrica compreende a formação acadêmica, conscientização das gestantes, confronto da sociedade e criação de leis políticas públicas, dentre outras ações.

\section{CONSIDERAÇÕES FINAIS}

De acordo com a literatura, foi importante o uso de fontes mais amplas de 2010 a 2020 para que esclarecessem os últimos 10 anos de violência obstétrica sofridos por mulheres durante o parto sendo eles verbalmente, fisicamente, procedimentos inviáveis e sem fundamento para tal, além disso, observou-se a 
violação do direito de escolha a acompanhante, o que nos possibilitou uma maior abrangência do presente estudo. Os resultados obtidos por meio desta revisão demonstram a indispensabilidade de viabilizar um ambiente mais adequado para o oferecimento da assistência desde o parto até o nascimento, onde seja possível ter acesso aos direitos e garantias estabelecidos às usuárias do serviço de saúde.

\section{REFERÊNCIAS}

1. ANDRADE BP, AGGIO CM. Violência Obstétrica: a Dor que Cala. Anais do III Simpósio Gênero e Políticas Públicas, 2014; 1 -7.

2. ANDRADE PON, et al. Fatores Associados à Violência Obstétrica na Assistência ao Parto Vaginal em uma Maternidade de Alta Complexidade em Recife, Pernambuco. Revista Brasileira de Saúde Materno Infantil, 2016; 16(1): 29-37.

3. ASSIS JF. Interseccionalidade, Rascimo Institucional e Direitos Humanos: Compreensões à Violência Obstétrica. Revista Serviço Social Sociedade, 2018, 133: 547-565.

4. BRASIL. Lei n 11.108, de 7 de abril de 2005. Altera a Lei no 8.080, de 19 de setembro de 1990, para garantir as parturientes o direito à presença de acompanhante durante o trabalho de parto, parto e pós-parto imediato, no âmbito do Sistema Único de Saúde SUS. Disponível em: http://www3.dataprev.gov.br/sislex/paginas/42/2005/11108.htm. Acessado em: 4 de outubro de 2021

5. CAVALHEIRO EAM, et al. Violência obstétrica: revisão de literatura. Revista Artigos, 2021; 06: 1-7.

6. GUIMARÃES LBE, et al. Violência Obstétrica em Maternidades Públicas do Estado do Tocantins. Revista Estudos Feminino, 2018; 26(1): 1-11.

7. HAMMED W, AVAN BI. Women's Experiences of Mistreatment During Childbirth: A Comparative View of Home- And Facility-Based Births in Pakistan. Plos One, 2018; 13(3): 1-17.

8. JARDIM DMB, MODENA CM. Obstetric Violence in the Daily Routine of Care and Its Characteristics. Revista LatinoAmericana Enfermagem, 2018; 26: 3069.

9. LANSKY S, et al. Violência Obstétrica: Influência da Exposição Sentidos do Nascer na Vivência das Gestantes. Revista de Ciência \& Saúde Coletiva, 2019; 24(8): 2811-2823.

10. LEAL MC, et al. Intervenções Obstétricas Durante o Trabalho de Parto e Parto em Mulheres Brasileiras de Risco Habitual. Caderno Saúde Pública, 2014; 30 (Sup): S17-S47.

11. MARTINS AC, BARROS GM. Parirás na Dor? Revisão Integrativa da Violência Obstétrica em Unidades Públicas Brasileiras. Revista Dor, 2016; 17(3): 215-8.

12. MARQUES GM, NASCIMENTO DZ. Alternativas que contribuem para a redução da violência obstétrica.. Revista Ciência Saúde Coletiva, 2019.

13. MEDEIROS NCM, et al. Violência obstétrica: Percepções Acerca do Parto Normal. Temas em Saúde, 2016; 16(3): 2447-2131.

14. MENDES, KDD, et al. Revisão Integrativa: Método de Pesquisa Para a Incorporação de Evidências da Saúde e na Enfermagem. Revista Texto \& contexto enfermagem, 2008; 17(4): 758-64

15. MENEZES FR, et al. O Olhar de Residentes em Enfermagem Obstétrica para o Contexto da Violência Obstétrica nas Instituições. Revista Interface-Comunicação, Saúde, Educação, 2019; 24: 180-664.

16. MINISTÉRIO DA SAÚDE. Portaria no 569 , de $1^{\circ}$ de junho de 2000 - Institui o Programa de Humanização no Pré-natal e Nascimento no âmbito do Sistema Único de Saúde. Brasil, 2000. Disponível em: portaria-20n-c2-ba-20569-2c-20de201-c2-ba-20de-20junho-20de-202000.pdf. Acessado em: 4 de outubro de 2021

17. OLIVEIRA VJ e PENNA C.M. Discussing Obstetric Violence Through the Voices of Women and Health Professionals. Revista Texto contexto - enfermagem, 2017; 26(2): 1-10

18. ORGANIZAÇÃO MUNDIAL DA SAÚDE (OMS). Prevenção e Eliminação de Abusos: Desrespeito e Maus Tratos Durante o Parto em Instituições de Saúde. 2014. Disponível em: https://apps. who.int. Acessado em: 29 de setembro de 2021.

19. PALHARINI LA. Autonomia Para Quem? O Discurso Médico Hegemônico Sobre a Violência Obstétrica no Brasil. Cadernos Pagu, 2017; 49: 1-37.

20. PAULA E, et al. Violência Obstétrica e o Atual Modelo Obstétrico, na Percepção dos Gestores em Saúde. Revista Texto Contexto Enfermagem, 2020; 19: 1-14.

21. RIBEIRO DO, et al. A Violência Obstétrica na Percepção nas Multíparas. Revista Gaúcha Enfermagem, 2020; 41 : 16.

22. RUSSO JA, NUCCI MF. Parindo no Paraíso: Parto Humanizado, Ocitocina e a Produção Corporal de Uma Nova Maternidade. Revista Interface comunicação Saúde Educação, 2020; 24: 1-14.

23. SAMPAIO J, et al. Um Corte na Alma: Como Parturientes e Doulas Significam a Violência Obstétrica que Experienciam. Revista Estudos Feministas, 2019; 27(3): 1-10.

24. SENA LM, TESSER CD. Violência Obstétrica no Brasil e o Ciberativismo de Mulheres Mães: Relato de Duas Experiências. Revista Interface comunicação Saúde Educação, 2017; 21(60): 209-20.

25. SENS MM, STAMM AMNF. Percepção dos Médicos Sobre a Violência Obstétrica na Sutil Dimensão da Relação Humana e Médico-Paciente. Revista Interface comunicação Saúde Educação (Botucatu), 2019; 23: 1-16.

26. SOUZA MT, et al. Revisão Integrativa: O que é e Como Fazer. Revista Einstein (São Paulo), 2010; (8): $102-106$.

27. TESSER CD, et al. Violência Obstétrica e Prevenção Quaternária: o que é e o que fazer. Revista Brasileira de Medicina de Família e Comunidade, 2015; 10(35): 1-12.

28. ZANARDO GLP, et al. Violência Obstétrica no Brasil: Uma Revisão Narrativa. Revista Psicologia Social, 2017; 29 : 1 11. 